\title{
Publicidad y competencia mediática para la educación ambiental en alumnos de primaria
}

\section{Advertising and media competence for environmental education in primary school students}

Mario Megías-Delgado es Técnico de investigación de la Universidad de Huelva (España) (mario.megias@dedu.uhu.es) (DD (https://orcid.org/0000-0002-2035-1479)

Patricia De-Casas-Moreno es Profesora Asociada de la Universidad Antonio de Nebrija (España) (pcasas@nebrija.es) (D) (https://orcid.org/0000-0003-1205-8106)

Gema Paramio-Pérez es Profesora Ayudante Doctor de la Universidad de Cádiz (España) (gema.paramio@uca.es) (iD (https://orcid.org/0000-0002-3359-1981)

Recibido: 2019-01-21 / Revisado: 2019-04-28 / Aceptado: 2019-05-17 / Publicado: 2019-07-01

\section{Resumen}

Los medios de comunicación y, sobre todo, la publicidad juegan un papel muy importante en la formación de la sociedad, especialmente, en el sector más joven. En este sentido, hay que reforzar los modelos pedagógicos en el aula para conseguir ciudadanos críticos. Por otro lado, el medio ambiente, en los últimos tiempos, se ha convertido en uno de los temas más populares de estudio. Por lo tanto, la presente investigación pretende arrojar luz sobre el uso de la publicidad para la enseñanza de la educación ambiental en el contexto de la Educación Primaria. Para ello, se ha llevado a cabo una investigación cualitativa a través de la observación participante y grupos focales. La muestra compuesta por cinco spots publicitarios relacionados con temas medioambientales y 75 alumnos de la granja escuela Huerto-Alegre de Granada, trata de esclarecer el nivel de conocimiento que poseen estos estudiantes sobre el objeto de estudio. Los principales resultados y conclusiones demuestran que a pesar de vincular problemas medio ambientales con los spots visionados existe una discontinuidad en los contextos familiar y escolar, incluso con ayuda del mediador, incidiendo en la necesidad de reforzar las competencias en la formación y conocimiento del alumnado.

Descriptores: Educación ambiental, publicidad, educomunicación, persuasión, menores, análisis cualitativo.

Forma sugerida de citar: Megías-Delgado, M., De-Casas-Moreno, P., \& Paramio-Pérez, G. (2019). Publicidad y competencia mediática para la educación ambiental en alumnos de primaria. Alteridad, 14(2), 220-230. https://doi.org/10.17163/alt.v14n2.2019.06 


\begin{abstract}
The media and, above all, advertising play a very important role in the formation of society, especially in the youth sector. In this sense, we must reinforce the pedagogical models in the classroom to get critical citizens. On the other hand, the environment, in recent years, has become one of the most popular topics of study. Therefore, this research aims to shed light on the use of advertising for the teaching of environmental education in the context of Primary Education. For this, qualitative research has been carried out through participant observation and focus groups. The sample consists of five
\end{abstract}

\section{Introducción y estado de la cuestión}

Los medios de comunicación se han alzado como un instrumento de persuasión esencial para la transformación social. En esta misma línea, el poder de los mensajes informativos, la manipulación y la sobresaturación de información, está causando nuevos fenómenos que deben ser estudiados como elementos prioritarios y con la mayor rigurosidad posible. En este punto, la publicidad cobra un rol influyente en la rutina diaria de las personas, que dedican gran parte de su tiempo a consumir información cada vez más personalizada por las marcas publicitarias, hecho que fomenta el consumo de productos determinados.

Por otro lado, cada vez es más candente la popularidad de temas mediáticos como el cambio climático y el auge de la publicidad medioambiental. Son muchos los retos a los que se enfrenta la educación medioambiental (EA). Entre ellos, la implantación de un sistema educativo que contemple esta temática como una materia prioritaria y no como un objetivo que se infiera de otras materias específicas como Ciencias de la Naturaleza o Ciencias Sociales. En esta línea, uno de los férreos escollos con los que se encuentra la EA en las escuelas es la dificultad de introducir programas educativos, que fomenten la concienciación del alumnado y la identificación de los contenidos didácticos con su entor- advertising spots related to environmental issues and 75 students of the Huerto-Alegre school farm in Granada, trying to clarify the level of knowledge that these students have about the object of study. The main results and conclusions show that in spite of linking environmental problems with the spots viewed there is a discontinuity in the family and school contexts, even with the help of the mediator, emphasizing the need to reinforce the competencies in the formation and knowledge of students.

Keywords: Environmental education, advertising, educommunication, persuasion, minors, qualitative analysis.

no cotidiano. El presente estudio exploratorio, pretende analizar la publicidad ambiental y la competencia mediática, como una herramienta eficaz para reducir la discontinuidad de ambas entre la familia y la escuela.

Hay que hacer hincapié en la repercusión de este tipo de temática, sobre todo, en términos educativos. De este modo, es necesario hacer alusión al documento internacional "Carta de la Tierra", donde se recoge en sus postulados sobre el alcance de los medios de comunicación. En su artículo 13.a., se señala la necesidad que posee la sociedad a recibir información sobre asuntos de carácter ambiental, así como a sus planes de desarrollo. Por su parte, el artículo 14.c., destaca la importancia de reforzar el rol de los medios de comunicación de masas como mediadores sociales sobre los actuales retos ecológicos. En conclusión, es importante empoderar educomunicativamente a los menores con el fin de desarrollar una correcta actitud crítica frente a los medios de comunicación. De este modo, se podrá comprobar y trabajar las diferentes posibilidades educativas de los anuncios existentes ligados a la EA.

\section{Perspectivas de la Educación Ambiental (EA)}

La preocupación sobre el medio ambiente es una tendencia cada vez más creciente. En este sentido, múltiples autores destacan aquellas problemáticas que diferencian los aspectos físicos- 
ambientales (el cambio climático, la desertización y deforestación, erosión del suelo, el aumento del gas carbónico, las alteraciones del ciclo del agua, la contaminación del aire, etc.), de los socio-ambientales destacando problemas como la superpoblación, la pobreza del Tercer Mundo, la economía y el medio ambiente, el problema de la energía, etc. (MoralesHernández, Caurín-Alonso, \& Souto-González, 2015; Castillo-González, 2018).

El concepto de medio ambiente abarca límites inimaginables, ya que posee una dimensión externa al propio sujeto y del mismo modo, comprende todo lo que le rodea a éste: factores bióticos y abióticos (aire, agua, animales, seres humanos, suelo, etc.), los elementos artificiales (artefactos tecnológicos, productos químicos, las ciudades, etc.) o elementos socio-culturales (lengua, hábitos, tradiciones, valores, etc.) y las relaciones del ser humano con respecto a estos elementos (Giordan, \& Souchon, 1999). Por lo tanto, se puede entender el concepto como "un todo donde se interrelaciona e interactúa todo lo natural y lo humano" (Jiménez, Yebra , \& Guerrero, 2015). A raíz de estas apreciaciones, es necesario instaurar y comprender las bases teóricas de la Educación Ambiental (EA).

En este sentido, en el Congreso Internacional de Educación y Formación sobre Medio Ambiente de Moscú, celebrado en el año 1987, se definió por primera vez este concepto

Proceso permanente en el cual los individuos y las comunidades adquieren conciencia de su medio y aprenden los conocimientos, los valores, las destrezas, la experiencia y también la determinación que les capacite para actuar, individual y colectivamente, en la resolución de los problemas ambientales presentes y futuros. (UNESCO-PNUMA, 1987, p. 3)

Hay que destacar que la Educación Ambiental, desarrolla actitudes proambientales frente a los problemas y retos ambientales de la actualidad (Marcote, \& Suárez, 2005; Castillo, 2010). Según Beck (2004) una EA efectiva será el resultado de reconducir todas las degradaciones del medio ambiente (efecto invernadero, lluvia ácida, destrucción de la capa de ozono, uso de pesticidas, radiación, pérdida de tierras vírgenes, erosión del suelo, demanda de aire y agua y la producción y gestión de residuos, entre otros) en todas sus dimensiones e interrelaciones. Sin embargo, es necesario comprender que no todos los colectivos sociales perciben de la misma manera los problemas ambientales, por lo que hay que buscar y plantear alternativas dispares para solucionar dichos inconvenientes. Por lo tanto, frente a esta panorámica, educadores/as ambientales y docentes juegan un papel esencial ya que son ellos/as quienes deberán ayudar a los múltiples sujetos a analizar la información, hacer buenos juicios y motivarlos para que participen activamente en la sociedad. Asimismo, deberán desarrollar e implementar programas pedagógicos para trabajar las habilidades y convertir la información en una práctica significativa (Flor, 2005; Torres-Rivera, Mesina-Calderón, Salamanca-Salazar, \& Sepúlveda-Sepúlveda, 2016; Páramo, 2017).

\section{La escuela frente a la EA. Razonamiento crítico y educación mediática}

Desde las propuestas de la Unesco en 1977 sobre la EA en las escuelas a través de la reforma progresiva de los programas de estudio, son numerosas las dificultades acontecidas. El tratamiento de la información solo se hace desde una larga maduración y desde una perspectiva fragmentaria que poco tiene que ver con el entorno de los discentes y que contrapone dos tipos de cultura: la superficial (divulgada por los medios de comunicación) y la escolar (dentro de programas disciplinares sin ningún tipo de relación con la realidad (Departamento de Ciencias, Educación Técnica y Ambiental, 1997).

De acuerdo con Sánchez y Sandoval (2012), la escuela debe promover en los contextos formales e informales la educación en 
medios. En este sentido, es necesario incluir a las familias para trabajar los mensajes publicitarios y sus dinámicas comunicativas. Este elemento es fundamental para reducir la disonancia en los procesos de enseñanza-aprendizaje y capacitar al alumnado a discernir los contenidos audiovisuales consiguiendo así un alumnado autónomo y con criterio propio.

Según Novo (2010), los principios básicos de la EA se pueden dividir en dos planos. Por un lado, el plano ético, donde se revisarán las posturas del hombre en relación con el entorno. En este punto, Stapp (1978) señala que deben establecerse vías efectivas como medio de acercamiento a los ejes nodales del comportamiento ambiental. Debe existir una vinculación entre lo que se hace (práctica) y con lo que sentimos al hacerlo (afectividad). De esta manera, se inculcan y asimilan valores, que van a modificar la relación del alumno con el medio. Por otro lado, hay que atender al plano conceptual, donde se estudia el modelo de concepto global del medio ambiente y se interrelacionan los aspectos naturales y los socioculturales. Asimismo, se consideran las relaciones e implicaciones sociales de los conceptos de energía y sus posibles usos para el desarrollo de una mejor calidad de vida.

Así pues, es necesario resaltar la complejidad que presenta la realidad, avalada por la EA, ya que es necesario la implicación de múltiples componentes para que sea efectiva. Asimismo, las emociones van a jugar un papel muy importante en su crecimiento, permitiendo la predicción de actitudes hacia el medio y cómo trabajar con él (Pooley, \& O'Connor, 2000; ColladoRuano, 2016). Por este motivo, es necesario la presentación de las características innatas del objeto de estudio para realizar una mejor contextualización sobre la materia: practicidad, la educación fomenta valores y conductas positivas para con el medio ambiente; moralidad, se trata de un proceso permanente en el que la sociedad se conciencia sobre el escenario estudiado; globalidad cognoscitiva, clarifica y armoniza las preocupaciones y valores (éticos, estéticos o eco- nómicos) de cada uno de los individuos y sociedades en relación con la percepción del medio ambiente; utilidad, busca la resolución práctica de los problemas, que afectan al medio ambiente $\mathrm{y}$ al ser humano; actualización, la EA necesita estar en constante reajuste de cara a los múltiples problemas medioambientales existentes; y humanizadora, derechos humanos y valores universales, aplicando estos principios se conseguirá la paz en la Tierra, así como la implantación de armonía con el medio físico-natural (NAAEE, 2000; Riera, Sansevero, \& Lúquez, 2017).

En definitiva, hay que prestar atención al conocimiento que se está desarrollando en materia ambiental, estableciéndose un proceso de aprendizaje permanente, enfrentándose la sociedad a los problemas ambientales que se les presenta y considerando las emociones como eje central de la Educación Ambiental, destacando la necesidad, por parte de los individuos, de poseer sus propio sistema de valores (Pérez, \& Rojas, 2016).

\section{Medios de comunicación, publicidad y EA}

Según Aznar-Minguet y Ull-Solis (2009) el nuevo ciudadano necesita de una educación integral para ser competente. El concepto de competencia es conocido como el conjunto de conocimientos, habilidades, destrezas y actitudes, requeridas en cada una de las circunstancias personales (Geli et al., 2004; Orden-Hoz, 2011; Scolari, 2018). De este modo, la formación de estos sujetos comprometidos con el medio ambiente, requiere una variación en los modelos interpretativos fundamentados en la relación entre el ser humano, el medio natural y el medio socio-cultural.

Sin duda, frente a la popularidad de los temas medioambientales, la influencia de los medios de comunicación, así como la sobresaturación de mensajes, han proliferado de manera alarmante. Esta explosión de información data de mediados de los años 70 en los Estados Unidos, instalándose posteriormente en el continente europeo. En España se puede destacar que en el 
año 2000, ya existían un total de 200 profesionales especializados en información ambiental, apareciendo en la misma fecha la Asociación de Periodistas de la Información Ambiental (APIA). En este sentido, aunque ya se ha expuesto el rol imperante que han cobrado este tipo de noticias en los medios de comunicación, es importante hacer hincapié en los estudios relacionados con la publicidad como objeto de estudio. Los primeros estudios que se realizaron en España relacionando la publicidad y el medio ambiente, fueron llevado a cabo por la Asociación Globalízate en el año 2005. Estos estudios analizaban la oferta y presentación de temáticas ambientales a través de anuncios publicitarios como spots de automóviles, contaminación atmosférica y ahorro energético, entre otros. Para poder estudiar este tipo de spots, la Asociación indicó una serie de criterios para poder valorar de forma adecuada los mensajes. Por un lado, positivos, se informa al receptor sobre la emisión que se produce, los tipos y cantidades que se generan, entre otros aspectos importantes sobre la contaminación. Por otro lado, indiferentes, se informa de algunos contenidos a través de la letra pequeña. Por último, negativos, en este tipo de contenidos existe una promoción de actitudes nocivas frente a la preservación del medio ambiente (Camana, \& Almeida, 2017; Mendoza, 2018).

Según Díez (1998), "si se analiza la publicidad, se demuestra que va dirigida en la mayor parte de los casos más a nuestras emociones que a proporcionarnos información". La utilización de esta estrategia es clave dentro de la publicidad ya que tanto el recuerdo de la marca como del mensaje que se transmite se asocia, se recuerda y se identifica mejor a través de un apego emocional (Baraybar-Fernández et al., 2017).

Por lo tanto, frente a este contexto presentado, es necesario trabajar y reforzar una alfabetización mediática, así como el desarrollo de una correcta actitud crítica. Esta toma un protagonismo esencial desde el año 2008 debido a la intervención del Parlamento Europeo, definiéndola como la aptitud para entender y evaluar las múltiples carac- terísticas de los medios de comunicación (Sánchez, \& Sandoval, 2012; López, \& Aguaded, 2015).

Según Ruiz y Conde (2002), la publicidad no debe ser el espejo de una realidad irresponsable con nuestro entorno recogiéndose a través de leyes reguladores, que sigue sin llevarse a cabo.

En la actualidad, el panorama mediático en temática medioambiental es desalentador, ya que existe una falta de profesionalización en el sector y además, una falta de interés por parte de las empresas anunciantes (Delmas, \& CuerelBurbano, 2011). Concienciar a la población con el objetivo de disminuir el actual deterioro medioambiental debería ser el eje central de la publicidad ambiental. Sin embargo, sigue siendo una utopía, ya que aquella que se genera es escasa, está marginada, politizada y muestra tintes catastrofistas. Ante esto, es palpable que los temas de medio ambiente se quedan siempre fuera de todos los informativos, a excepción de los grandes desastres naturales. Sin embargo, existe contenido ambiental en la programación de carácter comercial de la televisión, que está cultivando actitudes de carácter medioambiental.

En definitiva, la publicidad medioambiental exige unas necesidades específicas relacionadas con la instauración de una alfabetización crítica sobre la materia, ayudar al ciudadano a conocer y conservar los valores naturales, así como la necesidad de conocer los mecanismos de actuación ante la publicidad (Sabre, 2017).

\section{Material y métodos}

El desarrollo de la competencia ambiental vislumbra múltiples enfoques y metodologías de enseñanza-aprendizaje. La posibilidad de utilizar la publicidad como una herramienta de aprendizaje y apoyo a la educación medioambiental puede representar la posibilidad de unificar la discontinuidad actual producida entre el entorno del alumnado y la educación escolar.

El objetivo de la presente investigación es arrojar luz sobre el uso de esta herramienta para la enseñanza de la educación ambiental en el con- 
texto de la Educación Primaria. Se analizarán las competencias mediáticas del alumnado frente a la publicidad ambiental y su percepción sobre la vinculación con el entorno. Para ello, se ha contado con la participación de un educador ambiental especializado en comunicación, que actuó como mediador entre la información superficial presentada y los contenidos educativos.

El estudio presentado ha sido desarrollado con metodología cualitativa, para hacer un análisis profundo de la percepción subjetiva de la muestra frente al objeto de estudio. Las técnicas empleadas para el desarrollo de la misma, han sido la observación para la primera fase y el grupo focal y la entrevista semiestructurada (bajo consentimiento informado de los tutores legales), para la segunda fase, previa al análisis de la información.

La investigación se ha dividido en tres fases: selección de spots publicitarios, elaboración del guion de entrevista y puesta en marcha del grupo focal.

Fase I. Selección de spots: Se hizo una revisión exhaustiva de los anuncios emitidos en televisión durante 2007-2015, dando como resultado 27 spot seleccionados. La selección de este periodo de análisis responde a la posibilidad de que durante su vida hayan podido visualizarlo en diferentes medios. A través de la técnica de observación y aplicando los siguientes criterios de inclusión: temática medioambiental, familiaridad para los alumnos, duración (inferior a tres minutos para evita la pérdida de atención) y la relación de la empresa que se publicita con el medio ambiente; se seleccionaron finalmente 5 para llevar a cabo el grupo de discusión: Greenpeace 2010 y 2014, Coca-Cola 2011 y 2012, e Iberdrola 2014.

Es importante recalcar el papel que juega el medio ambiente en el spot, las acciones medioambientales positivas y negativas sustraídas del mensaje publicitario, y por último, el objetivo del spot o finalidad del mensaje a transmitir.

Fase II. Elaboración del guion de entrevista. En esta segunda fase se procedió a elaborar una batería de preguntas abiertas para dar respuesta a los objetivos del estudio. Tras varias cribas, los investigadores consensuaron aquellas que corresponderían a las categorías de análisis de la información posterior, dejando abierta la posibilidad de incluir otras que surgieran durante el proceso de desarrollo de los grupos de discusión y que pudieran ser de interés para el estudio.

Fase III. Elección de la muestra y desarrollo del grupo focal. La elección de la muestra fue intencional, y estuvo compuesta por un total de 75 alumnos de Educación Primaria, que participaron en una estancia educativo-ambiental de las que oferta granja escuela Huerto-Alegre, en Granada. Las edades de los sujetos estuvieron comprendida entre los 8 y 12 años, y pertenecían a tres centros educativos diferentes de distintas provincias (Béjar-Almería, Granada y Alhaurín de la Torre-Málaga).

Se realizaron cinco grupos focales con 15 alumnos en cada uno, distribuidos por rangos de edad $(8,9,10,11,12)$. En cada grupo estuvo presente un investigador y el mediador en educación ambiental.

La puesta en marcha de la actividad consistió en la visualización del Spot por parte de los alumnos y el desarrollo de la posterior discusión del mismo siguiendo el guion semiestructurado.

\section{Análisis y resultados}

La información extraída de los grupos de discusión se dividió por spots y se estructuró en cuatro categorías: el papel que juega el medio ambiente en el spot, las acciones medioambientales positivas sustraídas del mensaje publicitario, las acciones medioambientales negativas sustraídas del mensaje publicitario $y$, por último, el objetivo del spot o finalidad del mensaje a transmitir. El análisis fue realizado por dos investigadores de forma paralela a través del programa cualitativo Mxqda para garantizar la validez del estudio.

\section{Greenpeace-Lego}

Examinando el primer spot visualizado

"Lego y la empresa petrolífera Shell", se pueden 
destacar los siguientes resultados. En torno al papel que juega el medio ambiente, los alumnos señalan "petróleo" como el concepto más afín con este tema de estudio. Además, insisten en la gran agresión que sufre el medio ambiente con la extracción de este tipo de combustible. Esta visión del contexto, por lo general, está focalizada en el medio natural. Sin embargo, algunos alumnos señalan que del mismo modo, el medio social es importante frente a esta problemática.

En relación a las acciones visionadas y que respetan el medio ambiente, los estudiantes no han sabido identificar las acciones proambientales existentes en el spot, ni relacionarlas con su vida cotidiana. Por otro lado, la mayoría de los alumnos han afirmado, sin embargo, que las acciones de los seres humanos pueden ayudar o perjudicar al medio ambiente.

En relación a la pregunta sobre qué acciones se han podido visibilizar qué hagan daño al medio ambiente, los estudiantes en su mayoría, han atendido a la extracción del petróleo como acción negativa. De este modo, resaltan que produce tanto daño en el medio natural como en el social. Sin duda, este matiz ofrecido por los sujetos, muestra un hecho importante ya que han mostrado que son las personas las encargadas de realizar estas acciones y responsable de ellas.

En la misma línea de estudio y atendiendo a la reflexión sobre el mensaje del spot, como objetivo principal, los alumnos han indicado que se espera que con este mensaje publicitario se ayude a cuidar el medio natural. Una pequeña parte de la muestra también ha atendido al medio social. Sin embargo, solo un alumno ha detectado el verdadero fin del spot: el boicot a la empresa LEGO debido a su relación con la empresa petrolífera Shell. De este modo, se evidencia que el alumnado no ha entendido el verdadero mensaje.

El segundo spot visualizado, ha sido el de "Greenpeace: Océanos". Por lo tanto, frente al papel que juega el medio ambiente, se puede señalar que muchas de las respuestas han tendido a interpretar la inundación de ciertos espacios como algo malo para el medio natural, cuando es algo totalmente natural. Con esta curiosidad, múltiples alumnos se han atrevido a proponer acciones para cuidar el medio. En este sentido, en primer lugar, en relación a las acciones proambientales han incidido en que se debe cuidar el medio natural con el fin de mejorar la salud de la sociedad. Por su parte, aquellas acciones negativas más recalcadas son las causas del aumento de las mareas, no constatando el hecho de que estas sean naturales. Además, hay que destacar la implicación y seguridad que muestran en relación a la calidad del agua y el descongelamiento de los polos.

Para finalizar, en torno a la reflexión y mensaje transmitido por el spot, la totalidad el alumnado ha entendido como mensaje principal el cuidar el medio natural.

\section{Coca-Cola}

A continuación, atendiendo al tercer anuncio visualizado, se van a arrojar los resultados más destacados. En esta ocasión, se trata del spot de "Coca-Cola: Razones para crecer". Por lo tanto, atendiendo al papel que juega el medio ambiente en el contenido, la muestra ha señalado que las imágenes mostraban un medio bien cuidado, aunque ha habido algunos que han apuntado por su deterioro. La visión que ha predominado ha sido la del medio natural, sin embargo para algunos alumnos, está ha estado relacionada con el medio social.

En referencia a las acciones planteadas y que respeten el medio ambiente, las actitudes proambientales ofrecidas por los estudiantes han estado relacionadas con las normas acordadas en la presentación de la jornada de los alumnos durante su estancia en la granja escuela y no con lo visionado en el spot. De este modo, se puede destacar que para este ítem, a la hora del análisis y obtención de respuestas, no se han encontrado relación entre la información superficial y la escolar.

Según las acciones relacionadas con el daño al medio ambiente, los sujetos de la mues- 
tra han identificado la guerra y las acciones derivadas de ellas, como la creación de armas o lanzamiento de mísiles. Asimismo, también se ha incidido en otros hábitos nocivos como por ejemplo, el de tirar la basura al suelo.

Por último, ante la pregunta relacionada con el mensaje del spot, los resultados han sido contundentes, ya que el propio alumnado ha detectado que el principal fin del spot es el consumo de Coca-Cola.

En cuarto lugar, se ha analizado el spot "Día de la Paz: Coca-Cola". Por lo tanto, concerniente al papel que juega el medio ambiente, la muestra lo ha relacionado con el medio natural, relegando a un segundo plano al medio social y cultural. Asimismo, en el visionado también se pueden encontrar controversias en torno al cuidado del medio ambiente, ya que algunos señalan estar contaminado y otros no.

En referencias a las acciones desarrolladas para respetar el medio ambiente, preocupa el hecho de que la mayoría del alumnado no se haya percatado de ninguna acción. Además, algunos alumnos asocian las acciones proambientales a las normas impuestas en la granja escuela, como en el spot anterior. De igual forma, señalan que aquellas acciones nocivas son la circulación de vehículos, así como, las pintadas en los edificios. Estas respuestas son destacables, ya que son daños que se originan y afectan principalmente al medio social y cultural.

Por último, frente a la pregunta de reflexión sobre el fin del spot, los estudiantes han señalado que el objetivo del spot es el consumo de esta bebida aunque también se percatan de la posibilidad de ayudar y cuidar a los demás.

\section{Iberdrola}

Para finalizar con el quinto spot analizado, se ha visionado el anuncio de "Iberdrola Hoy", consiguiendo los siguientes datos. Por un lado, concerniente al rol del medio ambiente, se debe señalar que este spot es uno de los más complejos para los estudiantes, tanto por temática como por su discurso informativo. Por lo tanto, las respuestas obtenidas evidencian que el alumnado solamente ha tomado la interpretación del medio natural, aunque se hagan referencias al medio social a través de la aparición de ideas de construcciones.

En torno a las acciones que favorezcan y respeten el medio ambiente se puede destacar el uso de energías renovables. Asimismo, hacen énfasis de la política medioambiental de la empresa. Esta idea está recogida en el spot. Sin embargo, la muestra no llega a entender qué significa el concepto y qué consiste. Por su parte en las acciones desfavorables, las respuestas no han sido satisfactorias, ya que el alumnado no ha interpretado las acciones en contra del medio ambiente, sino que han mencionado todas aquellas que conocían de su vida cotidiana y, en algunos casos, han intentado relacionarlas con el spot.

Para finalizar, sobre la finalidad del spot, hay alumnos que han entendido que el mensaje es la contratación de los servicios de Iberdrola, mientras que otros, señalan que se trata de cuidar el medio ambiente cambiando nuestra forma de ser.

Para concluir con este apartado, se puede destacar como múltiples las apreciaciones e interpretaciones de los sujetos en relación al visionado de los spots. De este modo, se puede apreciar la necesidad de educar medioambientalmente y mediáticamente al alumnado, sobre todo, por la influencia de la ingente cantidad de información ofrecida a través de los medios de comunicación.

\section{Discusión y conclusiones}

Los medios de comunicación son una herramienta de influencia masiva en la actitud crítica de los espectadores. En este sentido, la publicidad se convierte en la mejor opción para mediar de forma positiva o negativa en la construcción de los idearios de la sociedad. A raíz de ello, surge la necesidad de educar mediáticamente a los más jóvenes, empoderándoles educomunicativamente con el fin de ser capaces por ellos mismos, interpretar y reflexionar en relación a 
la sobresaturación informativa. Frente a esto, las noticias y mensajes relacionados con el medio ambiente se han vuelto muy populares, así como, la Educación Ambiental. Por lo tanto, el presente estudio ha pretendido comprobar la validez de la publicidad, que se muestra a través de la televisión como recurso dentro de esta temática de Educación Ambiental.

Hay que destacar que tras realizar los análisis pertinentes, no se han alcanzado los resultados deseados, en el sentido que, los estudiantes no han sabido relacionar de forma correcta el concepto de medio ambiente en los spots visionados. Asimismo, se puede destacar la discontinuidad entre familia y escuela en el sentido que, la formación o conocimientos adquiridos en los diferentes contextos no se trabajan de forma paralela. De igual forma, no han conseguido relacionar los contenidos escolares, aun estando el mediador presente durante la prueba. De este modo, es necesario fomentar y trabajar este tipo de competencias en Educación Primaria. Del mismo modo, se parte de la urgencia de instaurar correctos modelos pedagógicos que ayuden a solventar estas carencias en el aula. También, para poder poner en marcha este tipo de recursos es necesario desarrollar una correcta alfabetización mediática entre los discentes a nivel publicitario con el fin de poder analizar correcta y críticamente un spot publicitario y descifrar los mensajes implícitos de este tipo de transmisión informativa.

Plantear esta alfabetización mediática y publicitaria supone un reto importante, ya que por desgracia no dispone de un espacio dentro de la educación formal. Por el contrario, los educadores deberán trabajar en un contexto de educación no formal, donde a la misma vez, se desarrollan y compiten múltiples temáticas a parte de la Educación Ambiental.

Sin duda, es importante educar para un consumo sostenible a través de la publicidad. Además, esta realidad se encuentra actualmente en una fase experimental. Sin embargo, hay que seguir apostando y trabajando por los conceptos y términos ligados al estudio para una mayor comprensión. Por ejemplo, durante la investigación se ha podido constatar que los alumnos identifican el medio ambiente con el medio natural, excluyendo el medio social, el medio cultural y las múltiples relaciones existentes, así como, el rol del hombre y sus acciones. Si no se establece este tipo de visión, difícilmente se podrá prestar atención en cómo el ser humano debe cambiar su relación con el medio ambiente.

Por otro lado, en relación a los resultados obtenidos tras el visionado de los spots, se puede destacar que cuidar el medio ambiente es una de las respuestas más reiterativas. Además, en todos aquellos anuncios que se desarrollaba la actividad dentro de un entorno urbano, los estudiantes relacionaban las actitudes proambientales con la limpieza del lugar. También, se puede hacer hincapié en algunas actitudes proambientales relacionadas con la inexistencia de la guerra o cualquier tipo de violencia. Sin duda, los sujetos han señalado diferentes acciones positivas y negativas en torno al medio ambiente, dejando constancia la repercusión de las acciones cotidianas del ser humano. Este tipo de respuestas y datos, confirman que a pesar de la ineficacia actual de la publicidad como recurso en Educación Ambiental, esta experiencia les ha servido para reflexionar sobre el objeto de estudio.

\section{Referencias bibliográficas}

Aznar-Minguet, P., \& Ull-Solís, A. (2009). La formación de competencias básicas para el desarrollo sostenible: el papel de la Universidad [Education in basic competences for sustainable development. The role of University]. Revista de Educación, (1), 219-237. (https://goo.gl/zU3Dbt).

Baraybar-Fernández, A., Baños-González, M., Barquero-Pérez, O., Goya-Esteban, R., \& De-la-Morena-Gómez, A. (2017). Evaluación de las respuestas emocionales a la publicidad televisiva desde el Neuromarketing [Evaluation of emotional responses to television advertising through 
Neuromarketing]. Comunicar, 52, 19-28. https://doi.org/10.3916/C52-2017-02

Beck, U. (2004). Poder y contrapoder en la era global: la nueva economía política mundial. Barcelona: Paidós.

Camana, Â., \& Almeida, J. (2017). Periodismo ambiental y los "ambientes posibles" [Environmental Journalism and possible "environments"]. Espacio abierto: cuaderno venezolano de sociología, 26(2), 27-40. (https://goo.gl/JjGRui).

Castillo, R. M. (2010). La importancia de la educación ambiental ante la problemática actual [The importance of environmental education in the face of current problems]. Revista Electrónica Educare, 14(1), 97-111. (https://goo.gl/12aoCW).

Castillo-González, M. (2018). Educación ambiental: un análisis crítico de su evolución hasta la actualidad [Environmental education: a critical analysis of its evolution to actuality]. Santiago (111) (https://goo.gl/dzCfoP).

Collado Ruano, J. (2016). Educación emocional: retos para alcanzar un desarrollo sostenible [Emotional education: challenges to achieve a sustainable development]. CIEG, 26, 27-46. (https://goo.gl/rcRzJc).

Delmas, M. A., \& Cuerel-Burbano, V. (2011). The drivers of greenwashing. California Management Review, 54(1), 64-87.

https://doi.org/10.1525/cmr.2011.54.1.64

Departamento de Ciencias, Educación Técnica y Ambiental (1997). Educación ambiental: principios de enseñanza aprendizaje. Bilbao: Los libros de la Catarata

Díez, M. (1998). La retórica del mensaje publicitario. Universidad de Oviedo: Servicio de Publicaciones.

Iniciativa Carta de la Tierra (2016). Carta de la Tierra. (https://goo.gl/6GxKE6).

Flor, J. I. (2005). Claves para la Educación Ambiental. Santander: Centro de Estudios Montañeses. (https://goo.gl/wXMWD7).

Geli, A. Ma., Junyent, M., \& Sánchez, S. (Ed.) (2004). Ambientalización curricular de los estudios superiores. Tomo III. Diagnóstico de la Ambientalización curricular de los estudios superiores. Universidad de Girona: Publicaciones de la Red-ACES.
Giordan, A., \& Souchon, C. (1999). La educación ambiental: guía práctica. Madrid: Diada.

Jiménez, M. J., Yebra, A., \& Guerrero, F. (2015). Las bases de la educación ambiental [The bases of environmental education]. Iniciación a la investigación, (1). (https://goo.gl/MBMFei).

López, L., \& Aguaded, M.l. (2015). Teaching Media Literacy in Colleges of Education and Communication. [La docencia sobre alfabetización mediática en las facultades de Educación y Comunicación]. Comunicar, 44, 187-195. https://doi.org/10.3916/C44-2015-20

Marcote, P. V., \& Suárez, P. A. (2005). Planteamiento de un marco teórico de la Educación Ambiental para un desarrollo sostenible [Proposing a theoretical frame of Environmental Education for sustainable development]. REEC: Revista Electrónica de Enseñanza de las Ciencias, 4(1). (https://goo.gl/nJf4Hv).

Mendoza, M. E. B. (2018). Comunicación, educación y medio ambiente: escenarios históricos de relación [Communication, education and environment: historical scenarios of relationship]. Criterio Libre Jurídico, 9(1). 73-96. (https://goo.gl//kBZNe).

Morales-Hernández, A. J., Caurín-Alonso, C., \& Souto-González, X. M. (2015). Percepción del mundo: mapas mentales y problemas socioambientales [Perception of the world: mental maps and socio-environmental problems]. Didáctica geográfica, (14), 91-108. (https://goo.gl/iGxx3U).

NAAEE (2000). Environmental Education Materials: Guidelines for Excellence. Workbook. Bridging Theory \& Practice. Rock Spring: North American Association for Environmental Education. (https://goo.gl/ujwRX9).

Novo, M. (2010). La educación ambiental, una genuina educación para el desarrollo sostenible [Environmental Education, a genuine education for sustainable development]. Revista de Educación, (1), 195-217. (https://goo.gl/h1on76).

Orden-Hoz, A. D. L. (2011). Reflexiones en torno a las competencias como objeto de evaluación en el ámbito educativo [Reflections on Competency Based Assesment in Education]. Revista electrónica de investigación educativa, 13(2), 1-21. (https://goo.gl/SfRqGg). 
Páramo, P. (2017). Reglas proambientales: una alternativa para disminuir la brecha entre el decir-hacer en la educación ambiental [Pro-environmental rules: An alternative for reducing the "say-do" gap in in environmental education]. Suma psicológica, 24(1), 42-58. http://dx.doi.org/10.1016/j.sumpsi.2016.11.001.

Pérez, F. G., \& Rojas, C. P. (2016). Ecopedagogía y ciudadanía planetaria. Madrid: De La Salle Ediciones.

Pooley, J. M., \& O'Connor, M. (2000). Environmental Education and Attitudes. Emotions and Beliefs are what is needed. Environment and behavior, 32(5), 711-723. https://doi.org/10.1177/0013916500325007

Riera, L., Sansevero, I., \& Lúquez, P. (2017). La educación ambiental: un reto pedagógico y científico del docente en la educación básica [Environmental Education: a pedagogical and scientific challenge of teachers in basic education]. Laurus 15(30), 392-406. (https://goo.gl/WLsAFj).

Ruiz, C. \& Conde, E. (2002). El uso del medio ambiente en la publicidad [The use of the Environment in Advertising]. Medio ambiente y comportamiento humano, 3(1), 89-101. (https://goo.gl/dXyvQG).

Sabre, M. E. (2017). Buenas prácticas para el uso del medioambiente en la publicidad. En Del verbo al bit (pp. 643-656). Sociedad Latina de Comunicación Social. (https://goo.gl/ MYaLCk).

Sánchez, J. \& Sandoval, Y. (2012). Claves para reconocer los niveles de lectura crítica audiovisual en el niño [Keys to recognizing the levels of critical audiovisual reading in Children]. Comunicar, 38, 113-120. http://dx.doi. org/10.3916/C38-2012-03-02

Scolari, C. A. (2018). Adolescentes, medios de comunicación y culturas colaborativas. Aprovechando las competencias transmedia de los jóvenes en el aula. Barcelona: Universitat Pompeu Fabra. (https://goo.gl/PSqXf1).

Stapp, W. (1978). Un modéle d'enseigment pour l'education environnementale. Perspectives, VIII(4). París: UNESCO. (https://goo.gl/38BpRH).

Torres-Rivera, L. B., Mesina-Calderón, N., SalamancaSalazar, B., \& Sepúlveda-Sepúlveda, C. (2016). Efectos de la enseñanza interdisciplinaria en la educación ambiental sobre los conocimientos, valores y actitudes ambientales de estudiantes de segundo ciclo básico (Los Ángeles, Región del Biobío, Chile) [Effect of interdisciplinary teaching of environmental education on knowledge, values and attitude of junior high school students (Los Ángeles City, Biobio Region, Chile)]. Revista Complutense de Educación, 27(3), 1139-1155. http://dx.doi.org/10.5209/rev_RCED.2016. v27.n3.47551 